\title{
Epithelial membrane protein 1 promotes glioblastoma progression through the PI3K/AKT/mTOR signaling pathway
}

\author{
LIFENG MIAO $^{1,2^{*}}$, ZHENG JIANG $^{1 *}$, JIWEI WANG ${ }^{1}$, NING YANG $^{1}$, QICHAO QI $^{1}$, WENJING ZHOU ${ }^{1}$, \\ ZICHAO FENG $^{1}$, WENJIE LI ${ }^{1}$, QING ZHANG ${ }^{1}$, BIN HUANG ${ }^{1}$, ANJING CHEN ${ }^{1}$, \\ DI ZHANG ${ }^{1}$, PENG ZHAO ${ }^{1}$ and XINGANG LI ${ }^{1}$ \\ ${ }^{1}$ Department of Neurosurgery, Qilu Hospital of Shandong University and Brain Science Research Institute, \\ Shandong University, Key Laboratory of Brain Functional Remodeling, Jinan, Shandong 250012; \\ ${ }^{2}$ Department of Neurosurgery, Dezhou People's Hospital, Dezhou, Shandong 253020, P.R. China
}

Received May 16, 2018; Accepted May 31, 2019

DOI: 10.3892/or.2019.7204

\begin{abstract}
Glioblastoma multiforme (GBM) is the most malignant intracranial tumor. Although the affected patients are usually treated with surgery combined with radiotherapy and chemotherapy, the median survival time for GBM patients is still approximately 12-14 months. Identifying the key molecular mechanisms and targets of GBM development may therefore lead to the development of improved therapies for GBM patients. In the present study, the clinical significance and potential function of epithelial membrane protein 1 (EMP1) in malignant gliomas were investigated. Increased EMP1 expression was associated with increasing tumor grade $(\mathrm{P}<0.001)$ and worse prognosis in patients $(\mathrm{P}<0.001)$ based on TCGA, Rembrandt and CGGA databases for human gliomas. In vitro, gene silencing of EMP1 in U87MG and P3 GBM (primary glioma) cells significantly inhibited tumor proliferation and invasion. In addition, it was revealed that activation of the PI3K/AKT/mTOR signaling pathway is the driving force of EMP1-promoted glioma progression. Finally, it was demonstrated, using an intracranial GBM animal model, that EMP1 knockdown significantly inhibits tumor growth in vivo and increases overall survival in tumor-bearing animals. Our research provides new insights into the molecular mechanisms underlying EMP1 knockdown-mediated inhibition of GBM cell invasion and raises the possibility that targeting of EMP1 may represent a promising strategy for the treatment of GBM.
\end{abstract}

Correspondence to: Dr Peng Zhao or Dr Xingang Li, Department of Neurosurgery, Qilu Hospital of Shandong University and Brain Science Research Institute, Shandong University, Key Laboratory of Brain Functional Remodeling, Shandong, 107 Wenhua Xi Road, Jinan, Shandong 250012, P.R. China

E-mail: drzhaopeng@163.com

E-mail:lixg@sdu.edu.cn

*Contributed equally

Key words: EMP1, glioma, proliferation, invasion, AKT

\section{Introduction}

Glioblastoma multiforme (GBM) is the most malignant primary human brain tumor. Tumors are characterized by a high proliferation rate and chemoresistance (1).

Although the combination of radiotherapy and chemotherapy has progressed after surgical resection, the 5-year survival rate of WHO grade IV glioblastoma is still lower than 5\% (2,3). Although significant progress has been made in understanding the molecular status of this type of tumor, there is still a need for new and effective therapeutic approaches (4-6).

Epithelial membrane protein 1 (EMP1) is a member of the EMP family that has been implicated as a cell junction protein on the plasma membrane (7). However, little is known about its specific functions and mechanisms. Recently, several members of the EMP family have been indicated to participate in cancer progression. EMP1 has been revealed to be a novel poor prognostic factor in pediatric leukemia and gastric carcinoma by regulating cell proliferation, migration and invasion, and was demonstrated to be involved in gefitinib resistance in lung cancer $(8,9)$. EMP1 was also identified as a novel MYC-interacting gene with cancer-related functions in GBM (10). However, the biological role of EMP1 in GBM remains unclear.

To the best of our knowledge, we are the first to determine the important role of EMP1 in GBM. In the present study, it was revealed that knockdown of EMP1 inhibited GBM cell proliferation, migration and invasion. In addition, it was determined that EMP1 is an independent predictor of poor prognosis in GBM patients. To sum up, our data indicated that EMP1 is a potential therapeutic target for the treatment of GBM.

\section{Materials and methods}

Ethical statement. All animal procedures were approved by the Institutional Animal Care and Use Committee (IACUC) of Shandong University (Jinan, China).

Database searches. The Cancer Genome Atlas (TCGA, http://cancergenome.nih.gov/), Rembrandt (http://www. 
betastasis.com/glioma/rembrandt/), and the Chinese Glioma Genome Atlas (CGGA, http://www.cgga.org.cn/) were mined for relevant molecular data.

Cell lines and cultures. Human glioblastoma cell lines U251 (cat. no. TCHu58), A172 (cat. no. TCHu171) and human glioblastoma of unknown origin cell line U87MG (cat. no. TCHu138, authentication was performed using STR Multi-Amplification Kit in Guangzhou Cellcook Biotech Co., Ltd.) were purchased from the Chinese Academy of Sciences Cell Bank (Shanghai, China). Human fibroblast glioblastoma cell line T98, primary human GBM biopsy xenograft propagated tumor cells P3 and normal human astrocytes were kindly provided by Professor Rolf Bjerkvig, University of Bergen (Bergen, Norway). Cells were cultured in Dulbecco's modified Eagle's medium (DMEM; cat. no. SH30022.01B; Thermo Fisher Scientific, Inc.) supplemented with $10 \%$ fetal bovine serum (cat. no. 10082147; Hyclone; GE Healthcare Life Sciences) in $5 \% \mathrm{CO}_{2}$ in a humidified incubator at $37^{\circ} \mathrm{C}$.

Immunohistochemical staining. Samples were fixed in $4 \%$ formalin at $20^{\circ} \mathrm{C}$ for $24 \mathrm{~h}$, paraffin-embedded and sectioned (4 $\mu \mathrm{m})$. After dewaxing and rehydration, sections were incubated with $0.01 \mathrm{M}$ citrate buffer at $95^{\circ} \mathrm{C}$ for $20 \mathrm{~min}$ for antigen retrieval. Endogenous peroxidase activity and non-specific antigen were blocked with $3 \%$ hydrogen peroxide and $10 \%$ normal goat serum (both from ZSGB-Bio; OriGene Technologies, Inc.), respectively, followed by primary antibody (EMP1; 1:100; cat. no. 63735; LifeSpan BioSciences) overnight at $4^{\circ} \mathrm{C}$. Sections were washed with phosphate-buffered saline (PBS), treated with goat anti-rabbit secondary antibody (cat. no. 1:200; cat. no. PV-9000; ZSGB-BIO), visualized with 3,3'-diaminobenzidine (DAB; both from ZSGB-Bio; OriGene Technologies, Inc.), and hematoxylin (Beyotime Institute of Biotechnology). Normal mouse serum served as a negative control.

shRNA transfections. Short hairpin (sh)-EMP1 (\#1: GTT TGTTAGCACCATTGCCAATGTTTCAAGAGAACATTG GCAATGGTGCTAACAAATTTTTT; \#2: GGTCTTTGG AAAAACTGTACCAATTCAAGAGATTGGTACAGTTTT TCCAAAGACCTTTTTT; \#3: GCCAGTGAAGATGCCC TCAAGACATTCAAGAGATGTCTTGAGGGCATCTTCA CTGGTTTTTT), were conjugated in the lentiviral vector of pLKO.1 with a puromycin resistant region (GenePharma). U87MG and P3 GBM cells were plated and infected with lentiviruses expressing sh-EMP1 for $24 \mathrm{~h}$, followed by puromycin selection ( $2 \mathrm{mg} / \mathrm{ml}$; Sigma-Aldrich; Merck KGaA). Western blotting was performed to verify knockdown efficiency and cells were allocated for different assays.

Cell viability assays. Cell viability was assessed using Cell Counting Kit-8 (CCK-8; cat. no. CK04-500; Dojindo Molecular Technologies, Inc.). Cells (1.0×10 4 cells/well) were seeded into 96 -well plates and incubated at $37^{\circ} \mathrm{C}$ overnight. After the desired treatment, the cells were incubated with $10 \mu \mathrm{l}$ of CKCK-8 in $100 \mu \mathrm{l}$ of serum-free DMEM for a further $4 \mathrm{~h}$ at $37^{\circ} \mathrm{C}$. The absorbance at $450 \mathrm{~nm}$ was measured using a microplate reader (Bio-Rad Laboratories, Inc.).
Western blot analysis. Cell lysates (20 $\mu \mathrm{g}$ protein) were subjected to western blot analysis, according to previously described protocols (11). Membranes were incubated with the following antibodies from Cell Signaling Technology: AKT (dilutions 1:1,000, cat. no. 9272), p-Akt (Ser473, dilution 1:1,000; cat. no. 4060), mTOR (dilution 1:1,000; cat. no. 2972), p-mTOR (Ser2448, dilution 1:1,000; cat. no. 2974), GAPDH (dilution 1:1,000; cat. no. 5174). Additional antibody was EMP1 (dilution 1:1,000; cat. no. 63735; LifeSpan BioSciences).

Cell migration and invasion assays. The wound healing assay was used to assess cell migration. U87MG and P3 GBM cells were seeded into 6-well flat bottom plates and incubated overnight at $37^{\circ} \mathrm{C}$. The cell-free space was created by scraping with a $200-\mu 1$ pipette tip. Wound closure areas were monitored at different time-points under a microscope and quantified using ImageJ software (National Institutes of Health). Cell invasion assays were performed in uncoated and Matrigel-coated Transwell chambers ( $8-\mu \mathrm{m}$ pore size inserts; Corning, Inc.). Cells $\left(2 \times 10^{4}\right)$ in medium containing $1 \%$ FBS $(200 \mu \mathrm{l})$ were seeded in the top chamber. The lower chamber was filled with medium containing $30 \%$ FBS $(600 \mu \mathrm{l})$. The cells that invaded the lower surface were fixed with $4 \%$ paraformaldehyde (Solarbio; Beijing, China) at $20^{\circ} \mathrm{C}$ for $15 \mathrm{~min}$, stained with $0.1 \%$ crystal violet (Solarbio) at $20^{\circ} \mathrm{C}$ for $15 \mathrm{~min}$ and counted under a bright field microscope. Images were captured from 5 random fields in each well and the number of cells was determined using Kodak MI SE 5.0 software (Carestream Health). Each experiment was repeated three times.

Intracranial xenograft model. Athymic mice (male; 4 weeks old; 20-30 g) were provided by Shanghai SLAC Laboratory Animal Co., Ltd. The mice were anesthetized with 5\% chloral hydrate and secured on a stereotactic frame. A longitudinal incision was made in the scalp and a 1-mm diameter hole was drilled $2.5 \mathrm{~mm}$ lateral to the bregma. Luciferase-stable P3 GBM cells $\left(2 \times 10^{5}\right)$ in $20 \mu \mathrm{l}$ of serum-free DMEM were implanted $2.5 \mathrm{~mm}$ into the right striatum using a Hamilton syringe. Mice were monitored by bioluminescence imaging every week. Briefly, mice were injected with $100 \mathrm{mg}$ of luciferin (Caliper; PerkinElmer, Inc.) while anesthetized with $3 \%$ isoflurane, followed by a cooled charge-coupled device camera (IVIS-200; Xenogen; Alameda, CA, USA). Bioluminescence values of tumors were quantitated using the Living Image 2.5 software package (Xenogen Corp.). Mice were sacrificed by $\mathrm{CO}_{2}$ inhalation and dislocation of neck after 30 days or when they developed neurological symptoms such as rotational behavior, reduced activity or displayed grooming and dome head. The brains were extracted, perfused with $4 \%$ paraformaldehyde in PBS and coronally sectioned for immunohistochemistry assays.

Statistical analysis. Three independent experiments were performed, and results were expressed as the mean \pm the standard deviation (SD). Data were compared using paired Student t-tests or one-way ANOVA followed by Bonferroni tests in GraphPad Prism 6 software (GraphPad Software, Inc.). P-values determined from different comparisons $<0.05$ were considered statistically significant and are indicated as follows: ${ }^{*} \mathrm{P}<0.05 ;{ }^{* *} \mathrm{P}<0.01 ;{ }^{* * *} \mathrm{P}<0.001$. 
A

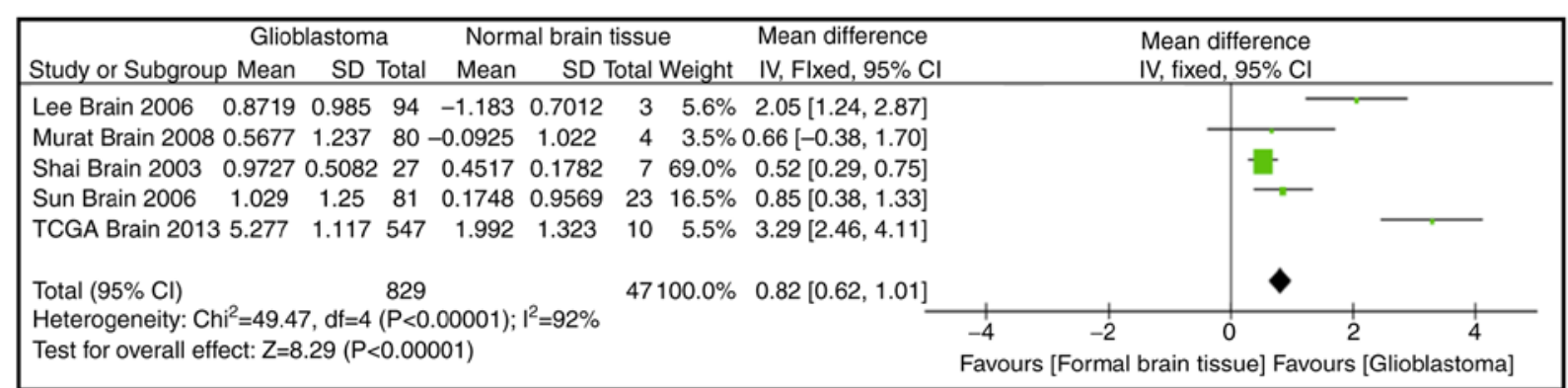
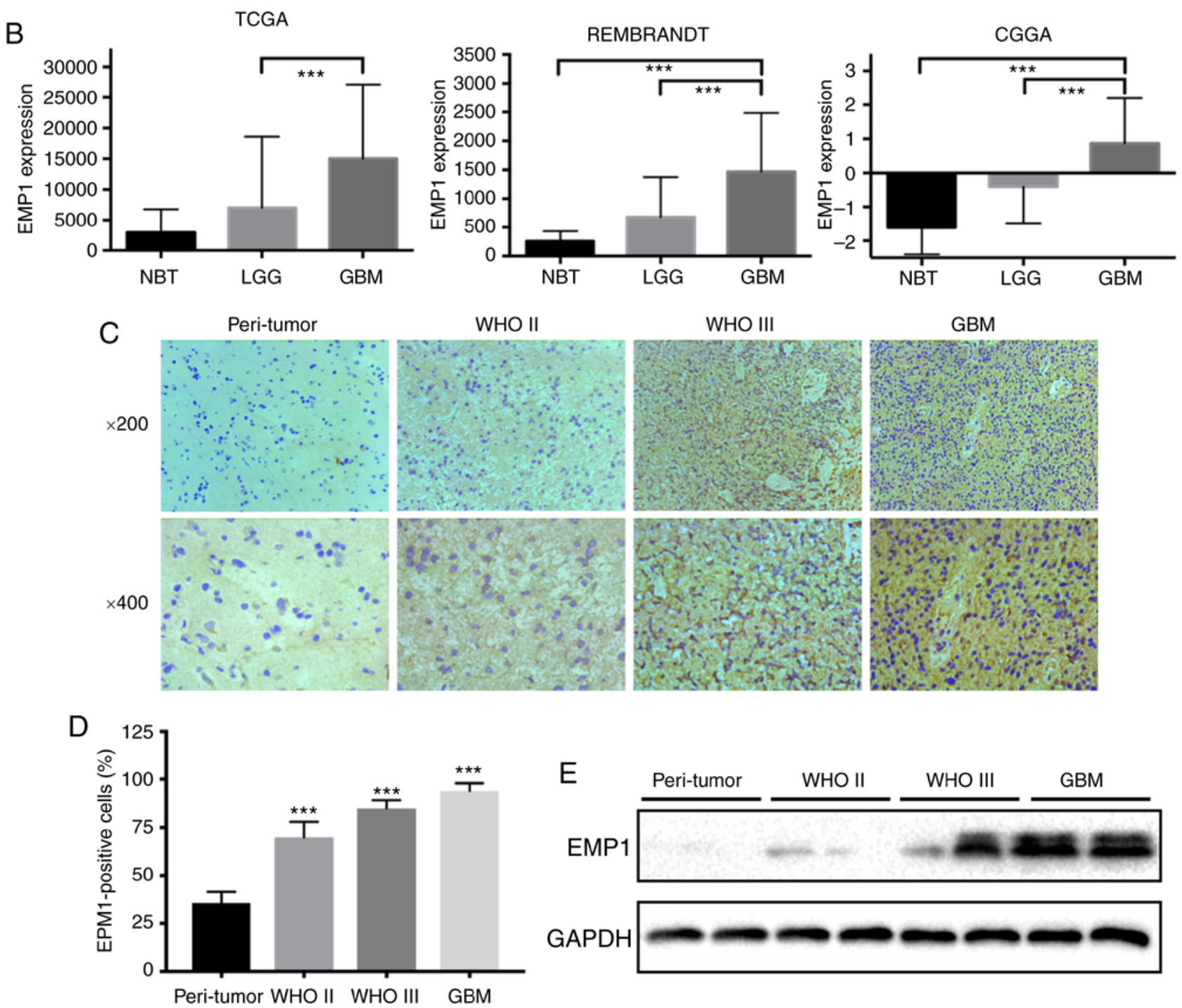

GAPDH

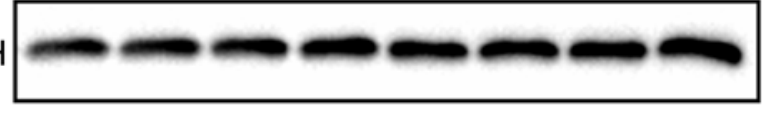

Figure 1. EMP1 expression is positively correlated with tumor grade. (A) Forest plot of EMP1 expression levels in glioblastoma (n=829) vs. non-neoplastic brain tissue samples $(\mathrm{n}=47)$ from the publicly available Oncomine datasets. The $\mathrm{x}$-axis is the standardized mean difference between glioblastoma and normal EMP1 expression based on a $\log _{2}$ scale. (B) mRNA expression levels of EMP1 as determined using TCGA, CGGA, and Rembrandt databases. (C) Representative images of IHC staining with anti-EMP1 antibody on human glioma and non-neoplastic brain tissue samples. Magnification x200, upper images; x400, lower images. (D) Graphical representation of scoring performed on IHC staining of glioma and non-neoplastic tissue samples for EMP1 levels. (E) Western blot analysis of EMP1 in lysates $(20 \mu \mathrm{g})$ prepared from different grades of human gliomas (WHO grades II-IV) and normal brain tissues. ${ }^{* * *} \mathrm{P}<0.001$ compared to the controls. GBM, glioblastoma; EMP1, epithelial membrane protein 1; TCGA, The Cancer Genome Atlas; CGGA, Chinese Glioma Genome Atlas.

\section{Results}

EMP1 expression is positively correlated with tumor grade. To begin, to determine whether EMP1 was differentially expressed between glioblastoma and normal tissues, microarray data from patient samples were extensively examined in the Oncomine database. A meta-analysis of five independent glioblastoma data sets including 829 human glioblastoma samples and 47 normal brain tissues revealed that EMP1 was significantly and consistently present in glioblastoma in all data sets (Fig. 1A). To further verify the level of EMP1 in normal brain tissues and different grade glioma tissues, the publicly available databases, The Cancer Genome Atlas (TCGA), Rembrandt, and the Chinese Glioma Genome 
Atlas (CGGA) were systematically retrieved and a relatively higher mRNA level of EMP1 was revealed in high grade gliomas compared to low grade gliomas $(\mathrm{P}<0.01$; Fig. 1B). Immunohistochemistry results revealed that increased EMP1 levels were associated with increased glioma grade. EMP1 was highly expressed in grade II/III astrocytoma and glioblastoma $(\mathrm{P}<0.01)$, whereas staining was weak or absent in peri-tumor tissues (Fig. 1C and D). Western blotting results corroborated these results. EMP1 protein levels were increased in glioma cases relative to peri-tumor tissues (Fig. 1E). Collectively, these results demonstrated that EMP1 levels were increased in glioma compared to normal brain tissues.

EMP1 expression is inversely associated with glioblastoma patient prognosis. The differences in expression levels of EMP1 in glioma and normal brain tissues prompted us to further investigate whether EMP1 can be used as a prognostic marker in glioma patients. Data from the TCGA, Rembrandt, and CGGA databases was used to determine the relationship between EMP1 levels and overall survival (OS) in glioma patients. Each sample was classified as EMP1-high expression if the signal was above the median expression for the population. These data revealed significant differences in OS and progression-free survival (PFS) between glioma patients with low EMP1 expression and those with high expression (Fig. 2).

Pathway analysis of EMPI and co-regulated genes. Next, to further understand the biological implications of EMP1 in gliomas, correlation analysis of EMP1 expression in whole genome gene profiling was performed in TCGA. The results revealed that 5,604 genes were correlated with EMP1 expression in TCGA database $(\mathrm{P}<0.01$; Fig. 3A). As illustrated in the volcano plot and heatmaps, these significant correlated genes were separated into positively correlated and negatively correlated genes (Fig. 3B and C). Subsequently, GO analysis revealed that EMP1 positively-correlated genes were strongly associated with biological processes including positive regulation of cell proliferation, negative regulation of apoptotic process, cell adhesion and extracellular matrix organization (Fig. 3D). In KEGG analysis, EMP1-correlated genes were enriched in pathways in cancer, especially in the PI3K/AKT signaling pathway (Fig. 3D).

Knockdown of EMPI inhibits proliferation, migration and invasion in glioma cells in vitro. To determine the biological roles in glioma, the expression level of EMP1 was first verified in several glioma cell lines. Western blots results confirmed that the expression levels of EMP1 protein were increased in several glioma cell lines, especially in P3 GBM, which was derived from a primary GBM through orthotopic passage in mice (12-14), and U87MG cells (Fig. 4A). shRNA targeting EMP1 lentiviral constructs were designed for stable knockdown of expression. EMP1 protein levels in U87MG and P3 GBM cells were significantly downregulated after infection with three different shRNAs against EMP1 compared to NC constructs, especially sh-EMP1-2 (Fig. 4B). Therefore, this shRNA was selected for the subsequent functional assays.

It was then determined whether EMP1 knockdown may be effective against GBM, using the cell viability assay, CCK-8.

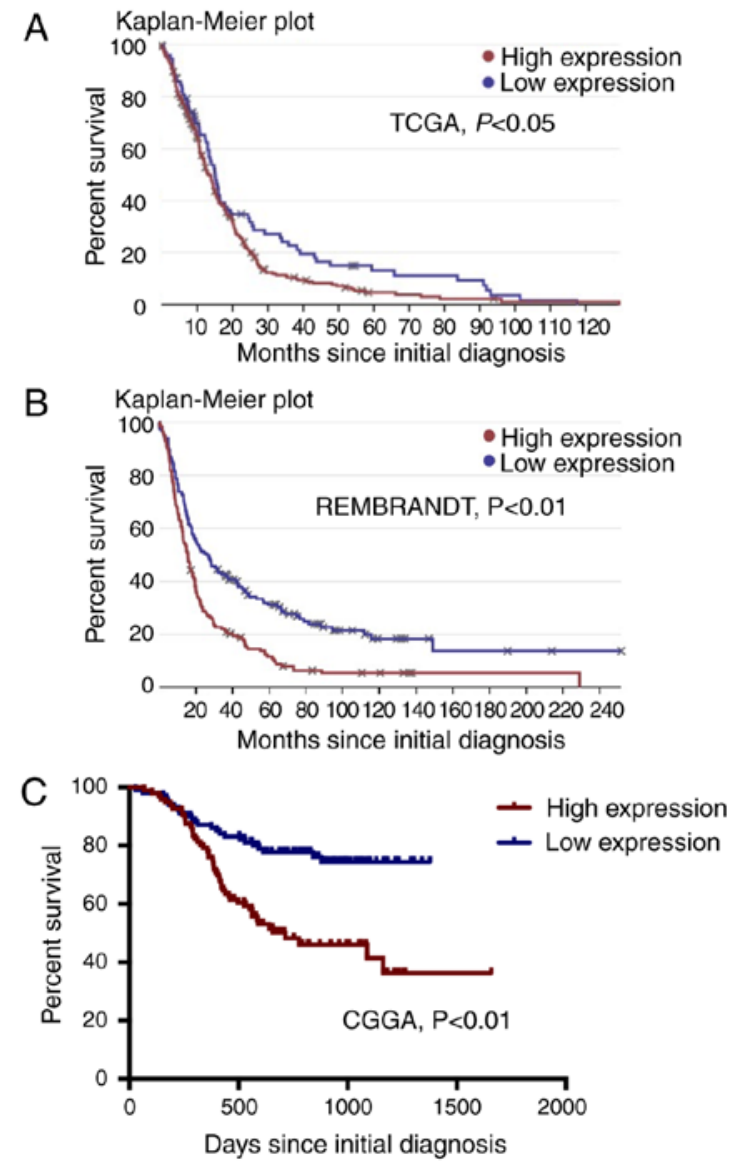

Figure 2. EMP1 expression is inversely associated with GBM patient prognosis. (A-C) OS analysis of EMP $1^{\text {low }}$ and EMP $1^{\text {high }}$ groups in GBM patients from TCGA, Rembrandt and CGGA databases. EMP1, epithelial membrane protein 1; GBM, glioblastoma; TCGA, The Cancer Genome Atlas; CGGA, Chinese Glioma Genome Atlas.

Knockdown of EMP1 led to significant decreases in cell viability in both U87MG and P3 GBM cells ( $\mathrm{P}<0.05$; Fig. 4C). Having observed a marked change in cell morphology and retraction of pseudopodia after knockdown of EMP1, a wound healing assay was used to examine whether knockdown of EMP1 affected migration in GBM cells. Knockdown of EMP1 led to a significant lower migratory rate both in U87MG and P3 GBM cells ( $\mathrm{P}<0.01$; Fig. 4D and E). Transwell analysis was further applied to assess the inhibitory effect of EMP1 knockdown on cell invasion. In order to mimic the extracellular matrix around glioma, U87GBM and P3 GBM cells were plated in the upper chambers of a Transwell system coated with Matrigel. The results revealed that the invasive ability of GBM cell was significantly decreased after knockdown of EMP1 (Fig. 4F and G).

EMP1 promotes human glioma progression through activation of the PI3K/AKT/mTOR signaling pathway. It is well known that abnormal activation of the PI3K/AKT/mTOR signaling pathway promotes tumorigenesis, and in KEGG analysis, EMP1-correlated genes were enriched in the PI3K/AKT signaling pathway (Fig. 3D). Therefore, phosphorylation status of AKT and mTOR proteins after knockdown of EMP1 was examined by western blotting to determine whether the observed responses could be due to a decrease in PI3K/AKT/mTOR 
A

P-value

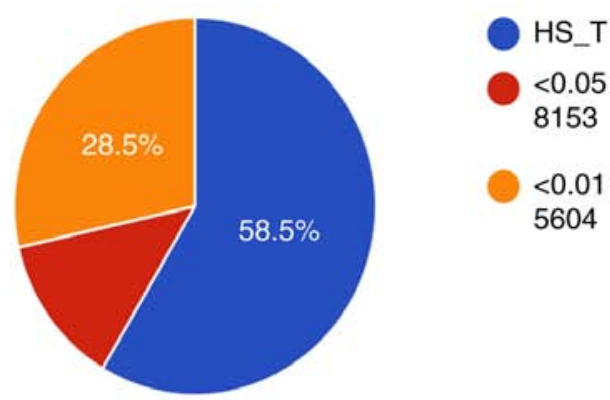

C

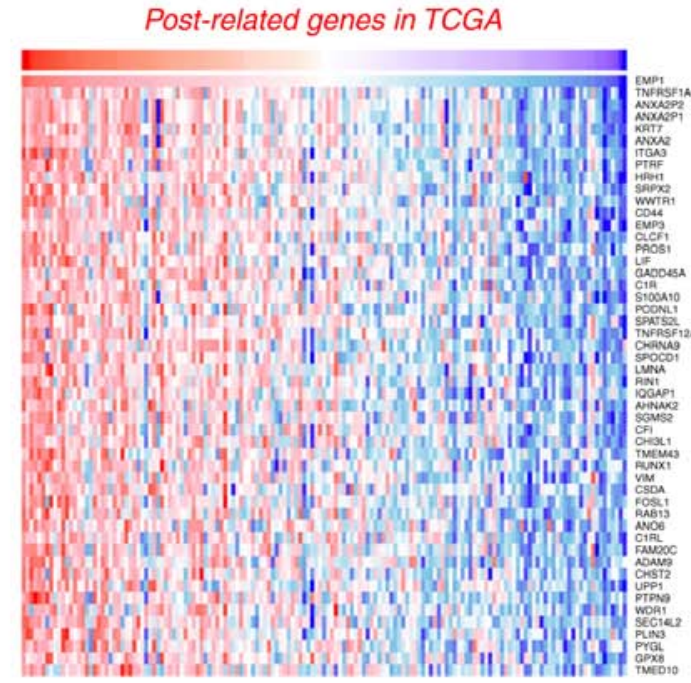

B

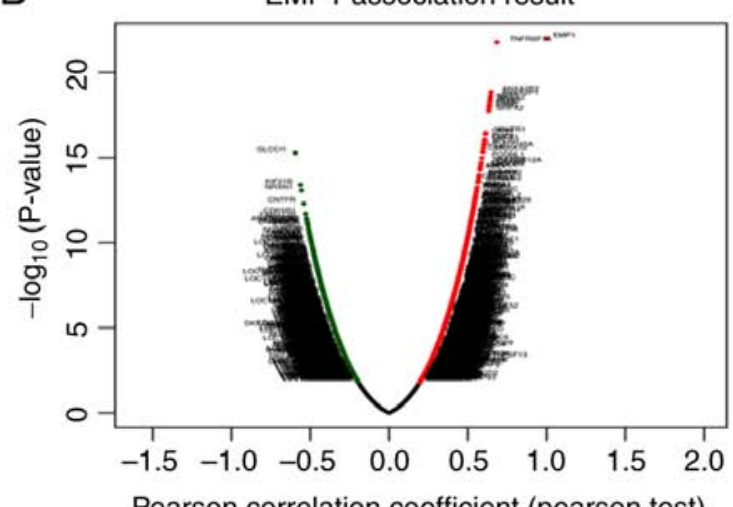

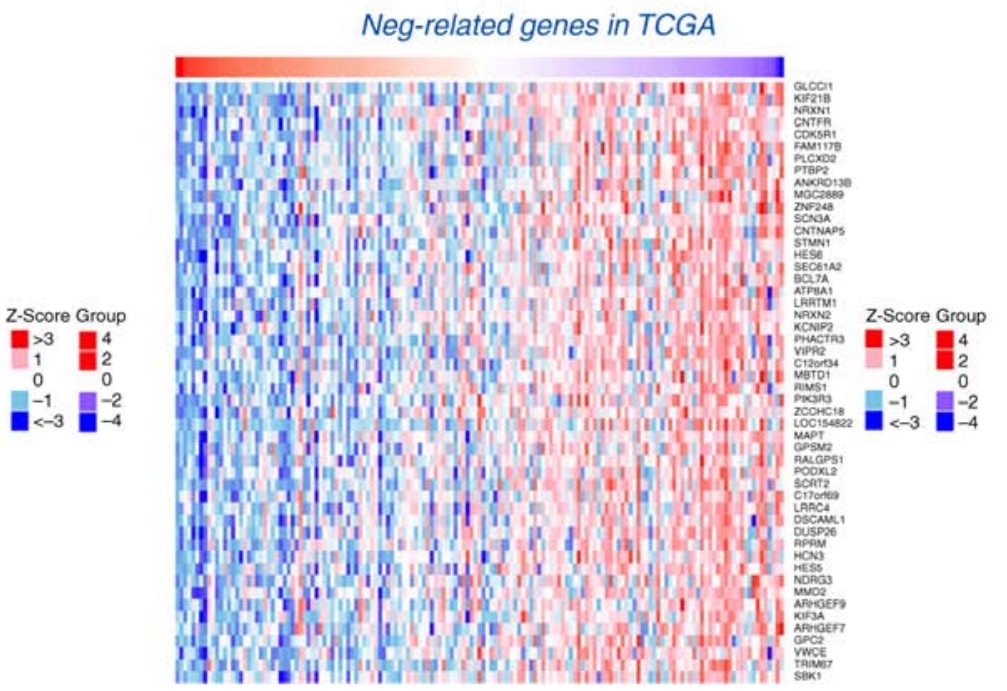

D

Biological processes

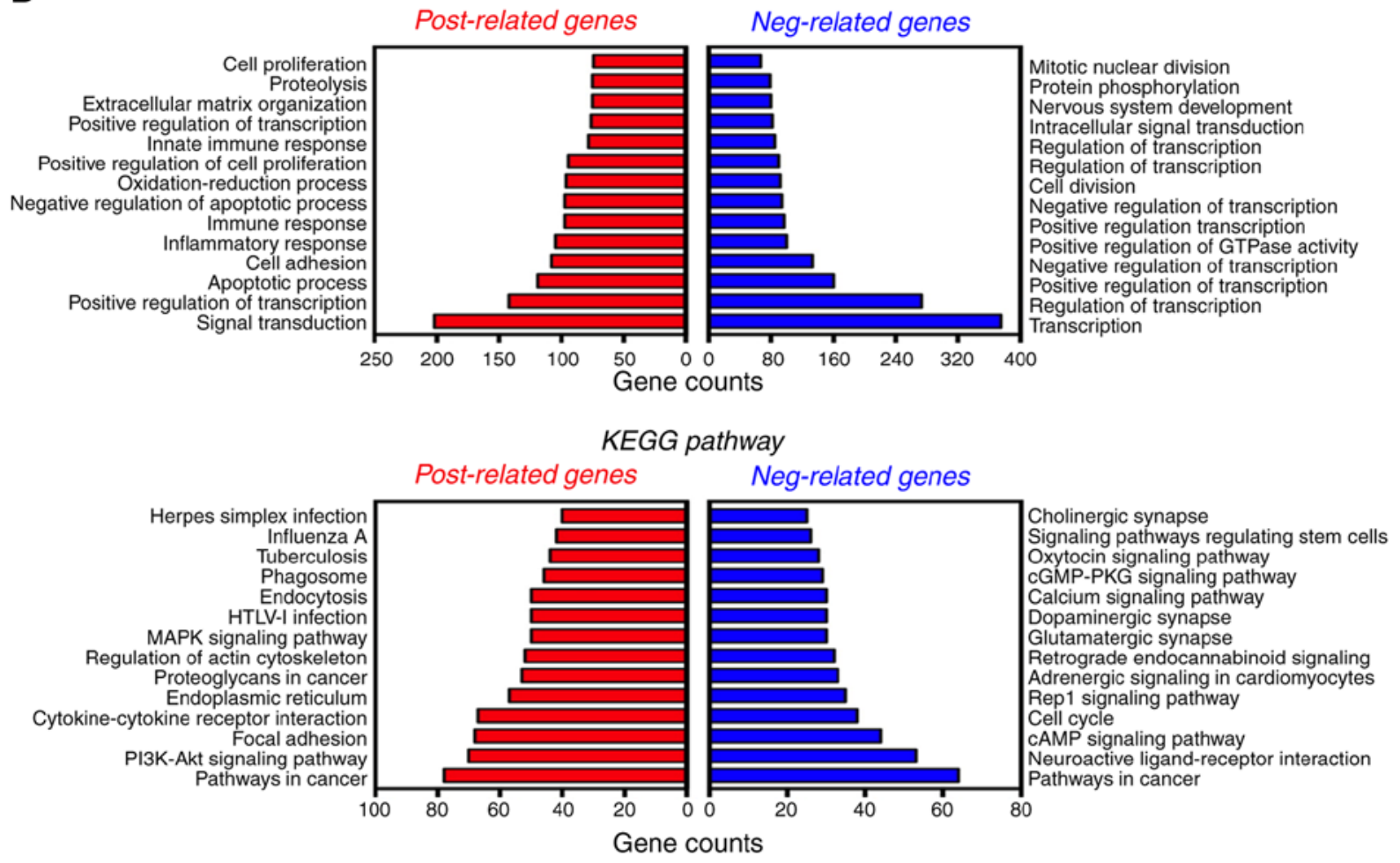

Figure 3. Pathway analysis of EMP1 and co-regulated genes. (A) Overview of genes correlated with EMP1 expression in TCGA database. (B) Volcano plot of genes correlated with EMP1 expression in TCGA database. (C) Correlation analysis using TCGA data revealing positively and negatively-correlated genes with EMP1 mRNA expression in human gliomas. (D) Biological processes and KEGG pathway analysis of the positively and negatively-correlated genes are illustrated. Potential functions and pathways are listed on the y-axis. EMP1, epithelial membrane protein 1; KEGG, Kyoto Encycopedia of Genes and Genomes. 
A

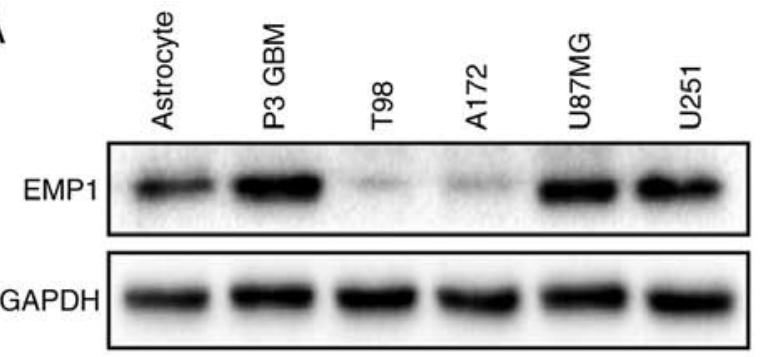

B
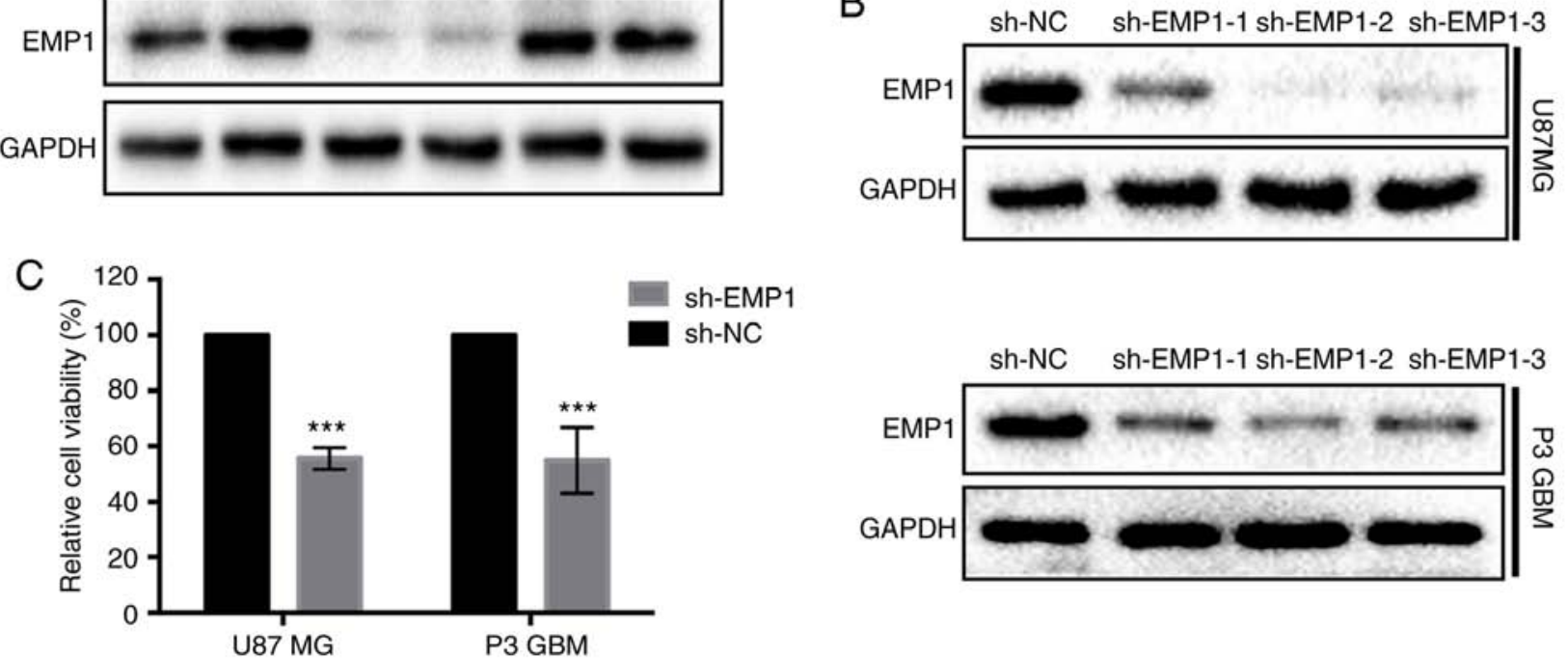

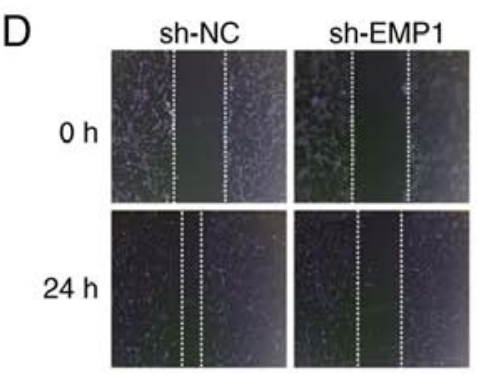

U87MG

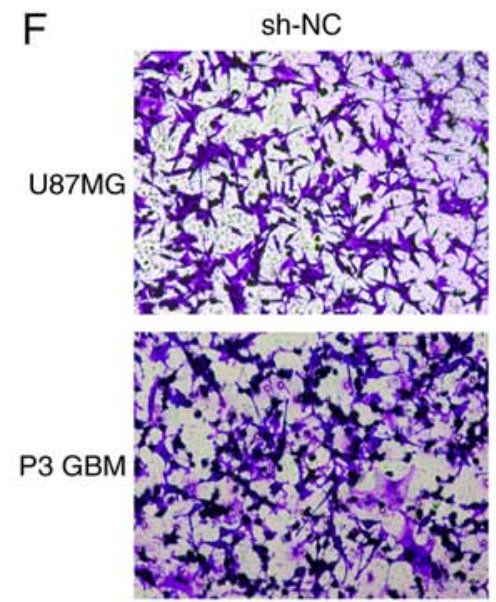

sh-NC

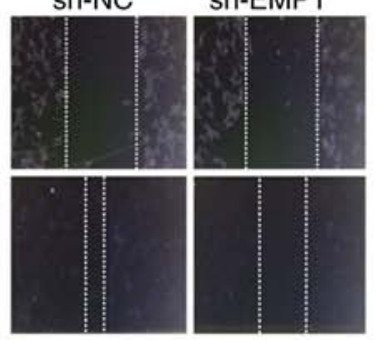

P3 GBM

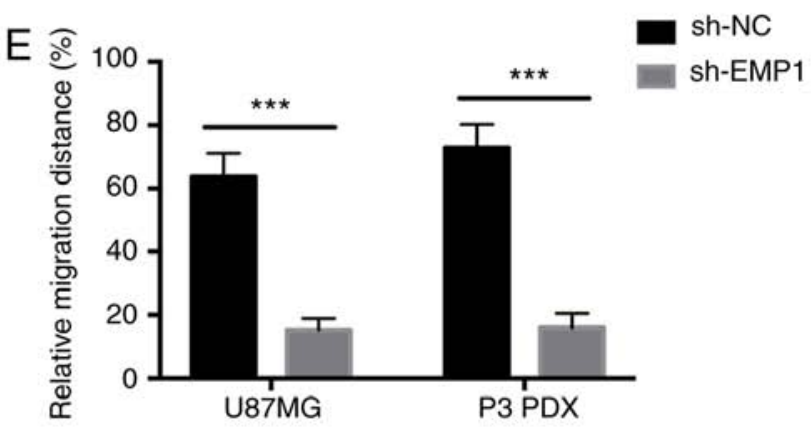

sh-EMP1

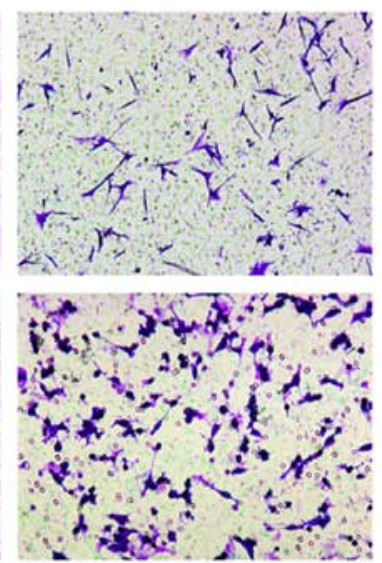

G

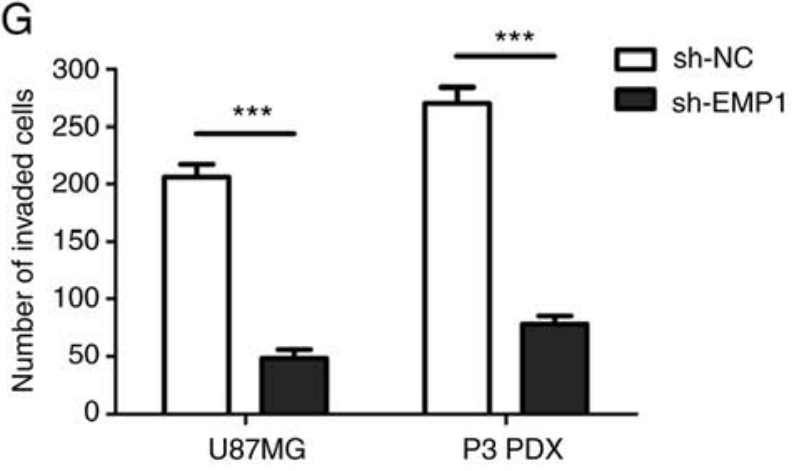

Figure 4. Knockdown of EMP1 inhibits proliferation, migration and invasion in glioma cells in vitro. (A) EMP1 expression in different types of human glioma cell lines. (B) Knockdown efficiency of EMP1 in U87MG and P3 GBM cells was determined by western blot analysis. (C) Viability as determined in CCK-8 assays performed on sh-NC/EMP1 U87MG and P3 GBM cells. Results are presented as a percentage (\%) relative to untreated cells. (D and E) The migratory ability and statistical results of the migratory rate of sh-NC/EMP1 U87MG and P3 GBM cells were evaluated by a wound healing assay. (F) Transwell invasion results of sh-NC/EMP1 U87MG and P3 GBM cells for $24 \mathrm{~h}$. (G) Statistical results of the invasive ratio of sh-NC/EMP1 U87MG and P3 GBM cells for $24 \mathrm{~h}$ in the Transwell assay. ${ }^{* * *} \mathrm{P}<0.001$ compared to sh-NC. EMP1, epithelial membrane protein 1; GBM, glioblastoma.

signaling. Phosphorylated AKT and mTOR decreased compared to sh-NC following knockdown of EMP1 in modified cells, demonstrating that the PI3K/AKT/mTOR signaling pathway was inhibited after EMP1 knockdown (Fig. 5A). After being treated simultaneously with the novel AKT activator SC79, the activation of the PI3K/AKT/mTOR signaling pathway in GBM cells was partially restored. SC79 increased phosphorylation of AKT and mTOR in EMP1-knockdown GBM cells, and therefore further confirmed PI3K/AKT/mTOR signaling as a molecular target for EMP1 knockdown of GBM growth (Fig. 5B). CCK-8 and Transwell invasion assays were repeated to assess whether SC79 could restore proliferation and 
A

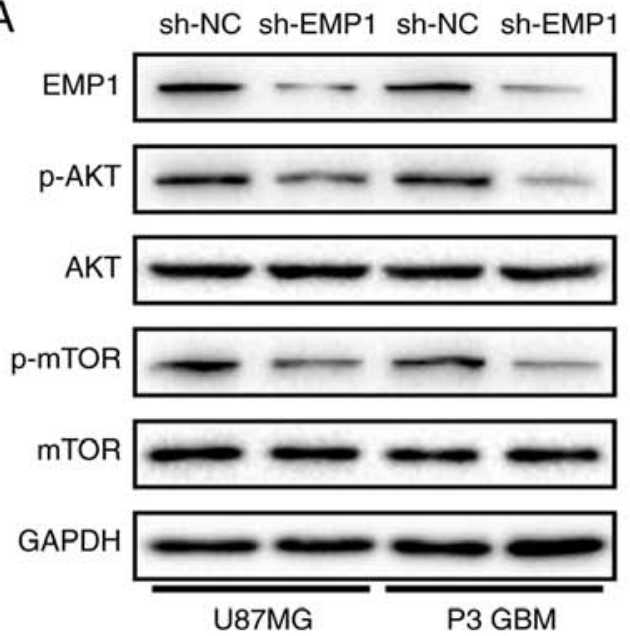

C

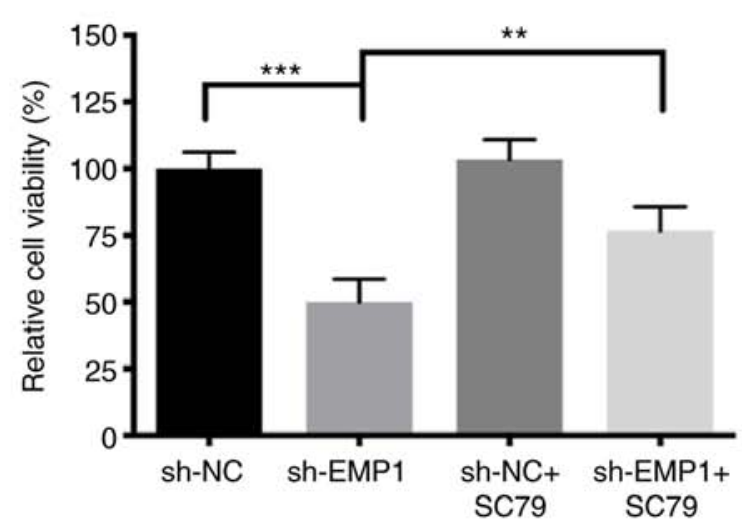

E

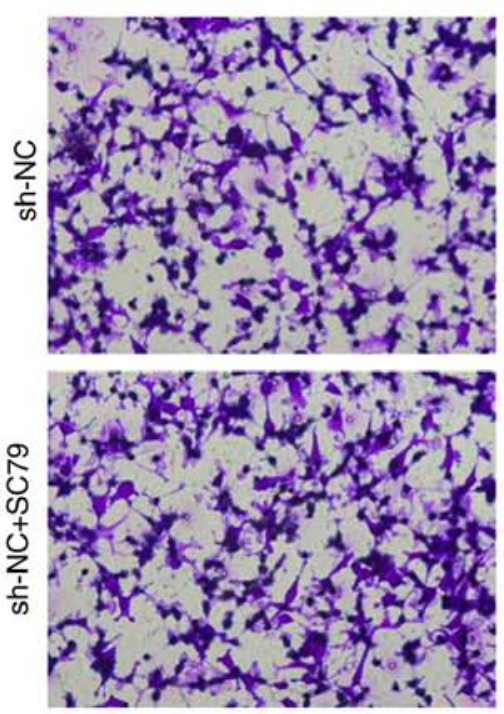

B
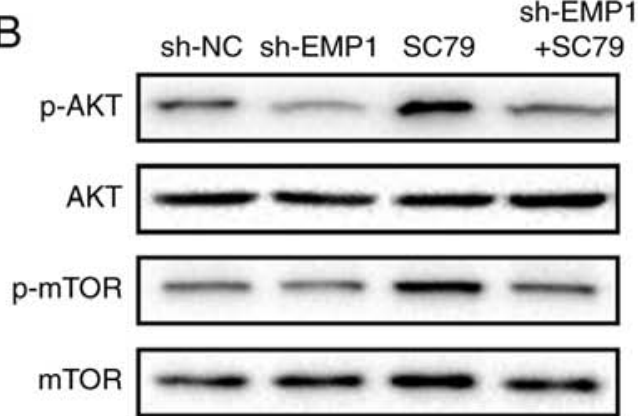

GAPDH

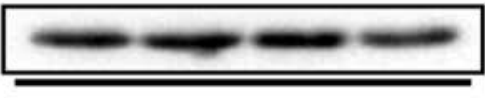

P3 GBM

D
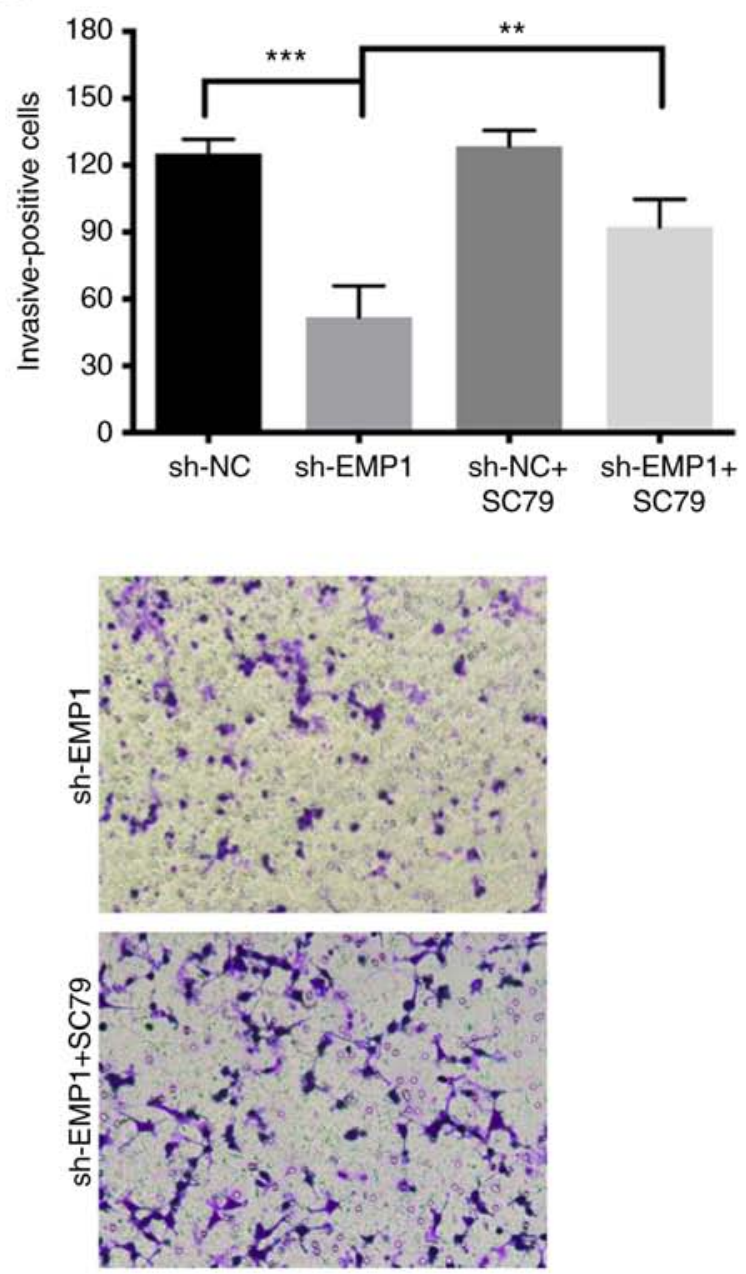

Figure 5. EMP1 promotes human glioma progression through activation of the PI3K/AKT/mTOR signaling pathway. (A) Western blot analysis of EMP1, p-AKT, AKT, p-mTOR, mTOR and GAPDH expression in sh-NC/EMP1 U87MG and P3 GBM cells. (B) Western blot analysis of p-AKT, AKT, p-mTOR, mTOR and GAPDH expression in sh-NC/EMP1 P3 GBM cells in the absence or presence of SC79 $(5 \mu \mathrm{g} / \mathrm{ml})$ for $24 \mathrm{~h}$. (C) Cell viability as determined in CCK-8 assays performed on sh-NC/EMP1 P3 GBM cells in the absence or presence of SC79 $(5 \mu \mathrm{g} / \mathrm{ml})$ for $24 \mathrm{~h}$. (D and E) Transwell results of sh-NC/EMP1 P3 GBM cells in the absence or presence of SC79 $(5 \mu \mathrm{g} / \mathrm{ml})$ for $24 \mathrm{~h}$. ${ }^{* *} \mathrm{P}<0.01$ and ${ }^{* * * *} \mathrm{P}<0.001$. EMP1, epithelial membrane protein 1; GBM, glioblastoma.

invasion in EMP1-knockdown GBM cells. The results demonstrated that SC79 increased proliferation ( $\sim 50$ vs. $70 \%$; sh-EMP1 vs. sh-EMP1+SC79; $\mathrm{P}<0.05)$ and invasion $(\sim 50$ vs. $90 \%$; sh-EMP1 vs. sh-EMP1+SC79; $\mathrm{P}<0.05)$ in EMP1-knockdown GBM cells (Fig. 5C-E).
EMPI enhances growth of GBM cells in vivo. Considering the heterogeneity of GBM, P3 GBM, which is an in vivo-propagated primary GBM tumor cell line, was applied to investigate EMP1 function. Athymic nude mice $(n=10)$ were implanted with luciferase-stable P3 cells in an intracranial tumor model and tumor 

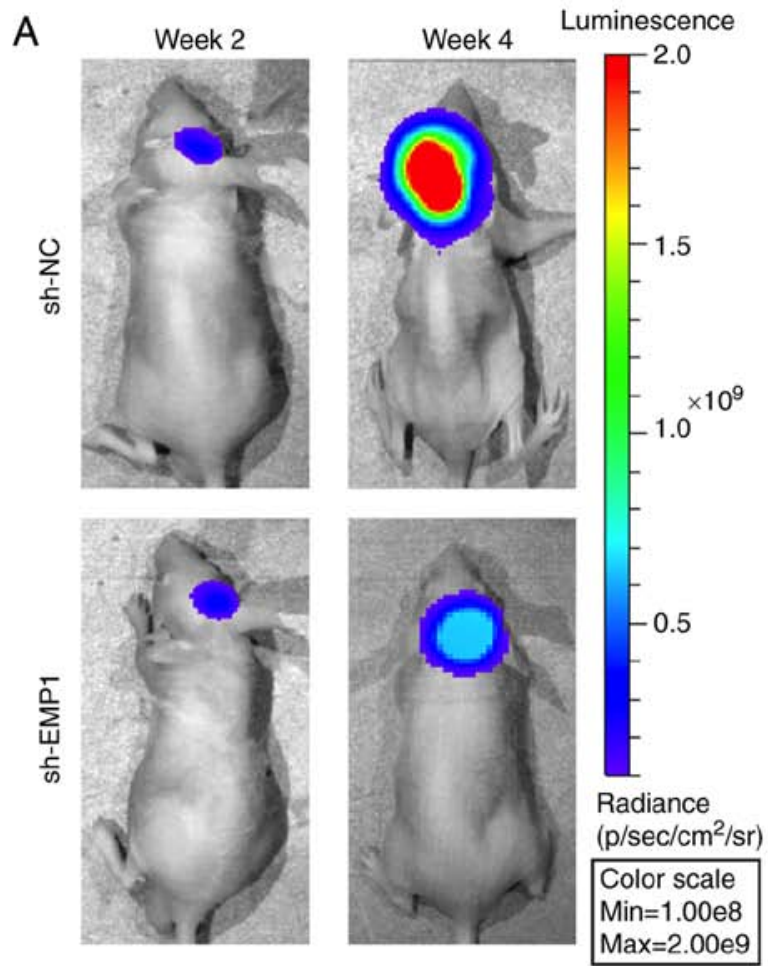

B
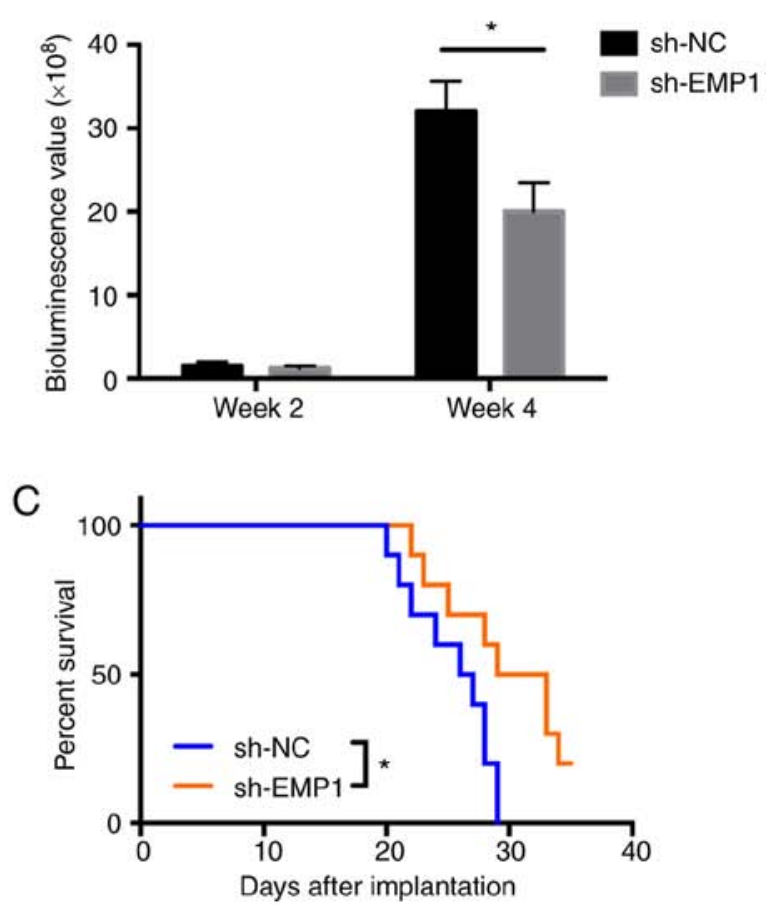

D

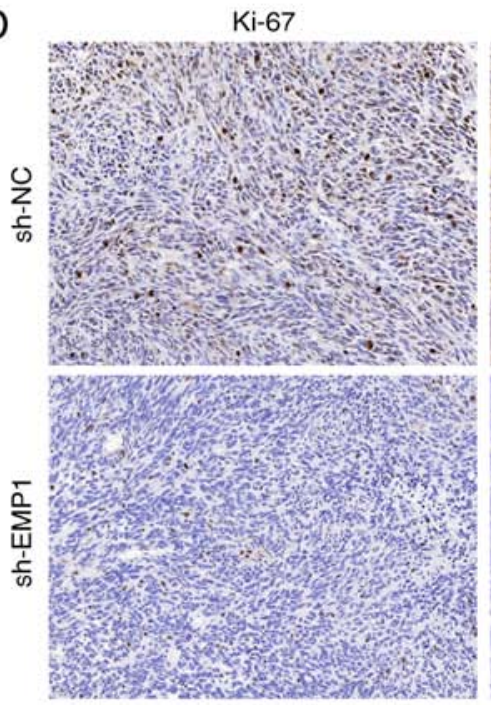

MMP2

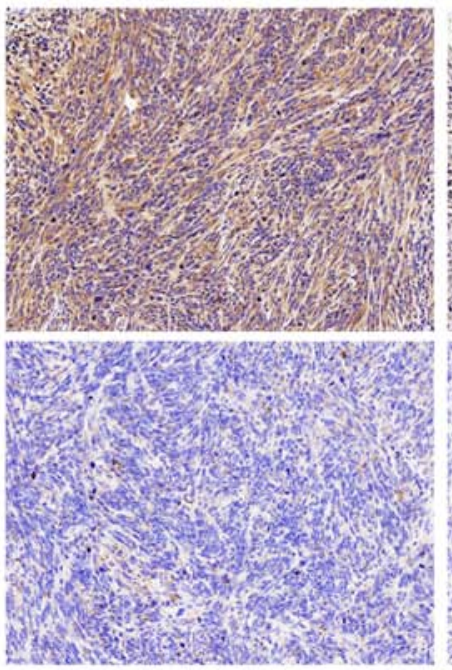

MMP9

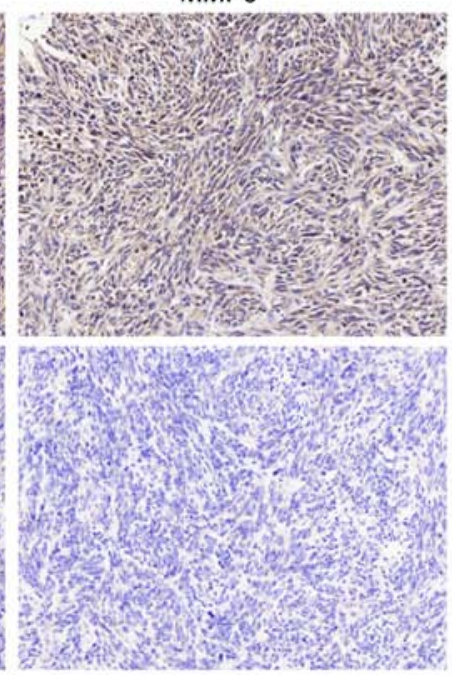

Figure 6. Knockdown of EMP1 suppresses GBM progression in vivo. (A) Luciferase-stable P3 GBM cells infected with lentiviruses expressing sh-NC or sh-EMP1 were orthotopically implanted into athymic nude mice, and tumor growth was monitored using the IVIS-200 imaging system for detection of bioluminescence. Bioluminescent signals were measured at days 14 and 28 after implantation. (B) Bioluminescence values as a function of time at days 14 and 28 to assess tumor growth. (C) Overall survival was determined by Kaplan-Meier survival curves, and a log-rank test was used to assess the statistical significance of the differences. (D) Images of immunohistochemical staining for Ki-67, MMP2 and MMP9 in tumors from each group as indicated. * $<0.05$. EMP1, epithelial membrane protein 1; GBM, glioblastoma.

growth was monitored over time using bioluminescence values. The results demonstrated that knockdown of EMP1 significantly reduced tumor growth $\left(\sim 35 \times 10^{8}\right.$ vs. $\sim 20 \times 10^{8}$ photons $/ \mathrm{sec}$, sh-NC vs. sh-EMP1; Fig. 6A and B). Kaplan-Meier analysis of the survival data demonstrated a statistically significant difference between sh-NC and sh-EMP1 mice ( $\mathrm{P}<0.05, \sim 29$ vs. $>32$ days, sh-NC vs. sh-EMP1; Fig. 6C). Immunohistochemistry (IHC) was performed on tissue sections from animals to examine proliferation and invasion. Ki-67, a marker for proliferation, MMP2 and MMP9, markers for invasion were decreased after EMP1 knockdown (Fig. 6D).

\section{Discussion}

Molecular-targeting therapy has become a promising therapeutic strategy for extending the survival time of cancer patients. Therefore, identifying novel therapeutic targets is critical for the design of more effective tumor specific strategies (15). Recently, several members of the EMP family have been indicated to participate in cancer progression (16-19). For instance, it has been reported that EMP1 is an oncogene of resistance to gefitinib in lung cancer, and it contributes to prednisolone resistance in patients with acute lymphoblastic leukemia. EMP2 was 
reported to be a biomarker in endometrial and ovarian cancer patients. Overexpression of EMP3 in breast cancer is significantly associated with high expression level of HER-2 which is one of the most important biomarkers of progression and metastasis-free survival for urothelial carcinoma of the upper urinary tract patients (19). Ramnarain et al revealed that the expression of EGFRvIII resulted in upregulation of a small group of genes including EMP1 in glioma cell lines (20). Bredel et al revealed that EMP1 is one of the novel MYC-responsive genes in gliomas (10). These studies indicated that EMP1 may be an oncogene in GBM, however, the biological role and underlying mechanism of EMP1 in GBM remains unclear.

In the present study, EMP1 was identified as a potential target of GBM molecular-targeting therapy. According to the mRNA microarray of TCGA, Rembrandt and CGGA, it was revealed that the mRNA level of EMP1 was increased in glioma compared to normal brain tissues. The IHC and western blot results of GBM or normal brain tissues further verified this view. Moreover, glioma patients with low EMP1 expression level had improved overall survival. Collectively, these data indicated that EMP1 could be associated with the malignancy of GBM and may serve as a novel prognostic indicator in clinical practice.

Abnormal cell proliferation, migration and invasion are hallmark characteristics of human gliomas. Many genetic changes lead to uncontrolled growth through dysregulation of proteins directly involved in cell cycle progression and cell invasion (21). GO analyses revealed that EMP1-assosiated genes exhibited significant enrichment mainly in processes related to cell proliferation, adhesion and extracellular matrix organization. The in vitro and in vivo data supported this analysis. EMP1 knockdown decreased the proliferation, migration and invasion in glioma cells and reduced tumor growth in orthotopic xenografts. Inhibition of cell proliferation can be the cause of senescence, apoptosis and autophagic cell death. Recent studies revealed that EMP1 also participated in nasopharyngeal and gastric cancer cell apoptosis $(9,22)$. Therefore, the cross-talk between EMP1 and senescence, apoptosis and autophagic cell death requires further exploration.

Glioma progression is a dynamic process in which the PI3K/AKT signaling pathway is a key event driving abnormal proliferation, differentiation and invasion of tumor cells (23). Mutations in the PTEN tumor-suppressor gene, a key regulator of the PI3K/AKT signaling pathway, lead to misaligned pathways in tumor cells. Results of KEGG analysis revealed that EMP1-associated upregulated genes were mainly enriched in the PI3K-AKT pathway. In the present in vitro study, the expression levels of p-AKT and p-mTOR were both decreased in EMP1-knockdown GBM cells. However, in the presence of an AKT activator, SC79, p-AKT and p-mTOR levels as well as functional activities including proliferation, migration, and invasion were partially restored. In conclusion, the $\mathrm{PI} 3 \mathrm{~K} / \mathrm{AKT} / \mathrm{mTOR}$ signaling pathway provides a molecular basis for the inhibition of tumor growth through EMP1 knockdown. However, the precise molecular mechanisms of the cross-talk between EMP1 and PI3K/AKT/mTOR signaling in gliomas require further investigation.

In summary, EMP1 facilitates proliferation, migration and invasion of GBM cells both in vitro and in vivo potentially by activation of the PI3K/AKT/mTOR signaling pathway. These results raise the possibility that targeting EMP1 may represent a promising strategy for the treatment of GBM.

\section{Acknowledgements}

The authors thank Professor Rolf Bjerkvig for providing human fibroblast glioblastoma cell line T98, primary human GBM biopsy xenograft propagated tumor cells P3 and normal human astrocytes, and Dr Janice Nigro for critical comments on the manuscript.

\section{Funding}

The present study was supported by the Natural Science Foundation of China Grants (81572487, 81402060 and 81472353), the Special Foundation for Taishan Scholars (ts20110814, tshw201502056 and tsqn20161067), the Department of Science and Technology of Shandong Province (2015ZDXX0801A01 and 2014kjhm0101), the Shandong Province Natural Science Foundation (ZR2014HM074), the Shandong Provincial Outstanding Medical Academic Professional Program, the Health and Family Planning Commission of Shandong province (2017WS673), the Fundamental Research Funds of Shandong University (2016JC019), the University of Bergen and the K.G. Jebsen Brain Tumor Research Centre.

\section{Availability of data and materials}

The datasets used during the present study are available from the corresponding author upon reasonable request.

\section{Authors' contributions}

$\mathrm{XL}$ and $\mathrm{PZ}$ conceived and designed the experiments; LM, ZJ, JW, NY, QQ, WZ and ZF performed the experiments; LM and ZJ analyzed the data; WL, QZ, BH, AC and DZ contributed to the reagents/materials/analysis tools. All authors were involved in the writing of the manuscript.

\section{Ethics approval and consent to participate}

All animal procedures were approved by the Institutional Animal Care and Use Committee (IACUC) of Shandong University (Jinan, China).

\section{Patient consent for publication}

Not applicable.

\section{Competing interests}

The authors declare that they have no competing interests.

\section{References}

1. Ricard D, Idbaih A, Ducray F, Lahutte M, Hoang-Xuan K and Delattre JY: Primary brain tumours in adults. Lancet 379: 1984-1996, 2012

2. Kalpathy-Cramer J, Gerstner ER, Emblem KE, Andronesi OC and Rosen B: Advanced magnetic resonance imaging of the physical processes in human glioblastoma. Cancer Res 74: 4622-4637, 2014. 
3. van den Bent M, Chinot OL and Cairncross JG: Recent developments in the molecular characterization and treatment of oligodendroglial tumors. Neuro Oncol 5: 128-138, 2003.

4. Garrido W, Rocha JD, Jaramillo C, Fernandez K, Oyarzun C, San Martin R and Quezada C: Chemoresistance in high-grade gliomas: Relevance of adenosine signalling in stem-like cells of glioblastoma multiforme. Curr Drug Targets 15: 931-942, 2014.

5. Lee CY: Strategies of temozolomide in future glioblastoma treatment. Onco Targets Ther 10: 265-270, 2017.

6. Uribe D, Torres A, Rocha JD, Niechi I, Oyarzún C, Sobrevia L, San Martín R and Quezada C: Multidrug resistance in glioblastoma stem-like cells: Role of the hypoxic microenvironment and adenosine signaling. Mol Aspects Med 55: 140-151, 2017.

7. Bangsow T, Baumann E, Bangsow C, Jaeger MH, Pelzer B Gruhn P, Wolf S, von Melchner H and Stanimirovic DB: The epithelial membrane protein 1 is a novel tight junction protein of the blood-brain barrier. J Cereb Blood Flow Metab 28 : 1249-1260, 2008.

8. Aries IM, Jerchel IS, van den Dungen RE, van den Berk LC, Boer JM, Horstmann MA, Escherich G, Pieters R and den Boer ML: EMP1, a novel poor prognostic factor in pediatric leukemia regulates prednisolone resistance, cell proliferation, migration and adhesion. Leukemia 28: 1828-1837, 2014.

9. Sun G, Zhao G, Lu Y, Wang Y and Yang C: Association of EMP1 with gastric carcinoma invasion, survival and prognosis. Int J Oncol 45: 1091-1098, 2014.

10. Bredel M, Bredel C, Juric D, Harsh GR, Vogel H, Recht LD and Sikic BI: Functional network analysis reveals extended gliomagenesis pathway maps and three novel MYC-interacting genes in human gliomas. Cancer Res 65: 8679-8689, 2005.

11. Wang J, Qi Q, Feng Z, Zhang X, Huang B, Chen A, Prestegarden L, $\mathrm{Li} X$ and Wang J: Berberine induces autophagy in glioblastoma by targeting the AMPK/mTOR/ULK1-pathway. Oncotarget 7: 66944-66958, 2016

12. Fack F, Espedal H, Keunen O, Golebiewska A, Obad N, Harter PN, Mittelbronn M, Bähr O, Weyerbrock A, Stuhr L, et al: Bevacizumab treatment induces metabolic adaptation toward anaerobic metabolism in glioblastomas. Acta Neuropathol 129: $115-131,2015$.

13. Fack F, Tardito S, Hochart G, Oudin A, Zheng L, Fritah S, Golebiewska A, Nazarov PV, Bernard A, Hau AC, et al: Altered metabolic landscape in IDH-mutant gliomas affects phospholipid, energy, and oxidative stress pathways. EMBO Mol Med 9: $1681-1695,2017$
14. Tardito S, Oudin A, Ahmed SU, Fack F, Keunen O, Zheng L, Miletic H, Sakariassen PØ, Weinstock A, Wagner A, et al: Glutamine synthetase activity fuels nucleotide biosynthesis and supports growth of glutamine-restricted glioblastoma. Nat Cell Biol 17: 1556-1568, 2015.

15. Rajesh Y, Pal I, Banik P, Chakraborty S, Borkar SA, Dey G, Mukherjee A and Mandal M: Insights into molecular therapy of glioma: Current challenges and next generation blueprint. Acta Pharmacol Sin 38: 591-613, 2017.

16. Hsieh YH, Hsieh SC, Lee CH, Yang SF, Cheng CW, Tang MJ, Lin CL, Lin CL and Chou RH: Targeting EMP3 suppresses proliferation and invasion of hepatocellular carcinoma cells through inactivation of PI3K/Akt pathway. Oncotarget 6: 34859-34874, 2015.

17. Lai S, Wang G, Cao X, Li Z, Hu J and Wang J: EMP-1 promotes tumorigenesis of NSCLC through PI3K/AKT pathway. J Huazhong Univ Sci Technolog Med Sci 32: 834-838, 2012.

18. Wang HT, Kong JP, Ding F, Wang XQ, Wang MR, Liu LX, Wu M and Liu ZH: Analysis of gene expression profile induced by EMP-1 in esophageal cancer cells using cDNA Microarray. World J Gastroenterol 9: 392-398, 2003.

19. Wang YW, Cheng HL, Ding YR, Chou LH and Chow NH: EMP1, EMP 2, and EMP3 as novel therapeutic targets in human cancer. Biochim Biophys Acta 1868: 199-211, 2017.

20. Ramnarain DB, Park S, Lee DY, Hatanpaa KJ, Scoggin SO, Otu H, Libermann TA, Raisanen JM, Ashfaq R, Wong ET, et al: Differential gene expression analysis reveals generation of an autocrine loop by a mutant epidermal growth factor receptor in glioma cells. Cancer Res 66: 867-874, 2006.

21. Hanahan D and Weinberg RA: Hallmarks of cancer: The next generation. Cell 144: 646-674, 2011.

22. Sun GG, Lu YF, Fu ZZ, Cheng YJ and Hu WN: EMP1 inhibits nasopharyngeal cancer cell growth and metastasis through induction apoptosis and angiogenesis. Tumour Biol 35: 3185-3193, 2014.

23. Manning BD and Toker A: AKT/PKB signaling: Navigating the network. Cell 169: 381-405, 2017.

This work is licensed under a Creative Commons Attribution-NonCommercial-NoDerivatives 4.0 International (CC BY-NC-ND 4.0) License. 